\title{
THE NEED OF TELEMETRY, TRACKING, COMMAND AND MONITORING SYSTEM IN THE GEOSTATIONARY SATELLITE
}

\author{
Baburajendra. $\mathbf{Y}^{1}$, CN. Udhay Kumar ${ }^{2}$, Shrihari $\mathrm{MR}^{3}$ \\ ${ }^{1}$ S.J.C. Institute of Technology, Chikkaballapur -562101 \\ ${ }^{2}$ S.J.C. Institute of Technology, Chikkaballapur - 562101 \\ ${ }^{3}$ S.J.C. Institute of Technology, Chikkaballapur - 562101
}

\begin{abstract}
The Telemetry system is used to transmit data obtained from multiple sensors on the satellite, whose purpose is to monitor the satellite via a telemetry link to the earth station. These systems are one part on the satellite and other at the controlling earth station. The tracking system is situated on this earth station and provides information regarding the range, elevation and azimuth angles of the satellite. In accordance with the telemetry data received from the satellite and orbital data received from the tracking system, the control system is used for the correction of the position and altitude of the satellite.It is also used to control the direction of the antenna and configuration of the communication system to suit current traffic requirements, and to handle switches on the satellite.
\end{abstract}

\section{INTRODUCTION}

For a communication satellite the TTC\&M is needed for its operation and the system sends this data to the earth's controlling station. There are various quality of controlling the task which are totally dedicated to a single work and it also its group members. The telemetry, tracking and control subsystem provides various means of communication between the space vehicle and itself. The main features of a satellite management are, to control the orbit and altitude of the satellite, observe the behavior of all the sensors and the subsystems on the satellite, and the measurement describes the satellite, subsystem by subsystem. These measurements involves magnitude which varies with different parameters, which vary in terms of voltage and current.

\section{TELEMETRY AND MONITORING SYSTEM}

The monitoring system functionality of collecting the data received from multiple sensors located in the satellites and after reading it, it sends these data to the earth's station. There might be hundreds of sensors on the satellite which are used to monitor various parameters such as pressure, voltage and current in the power conditioning unit, which acquires the voltage and currents in the communication electronics. The temperature of many subsystems should be kept within proposed limits as it is the critical parameter in communication systems. The data of sensor and the state of each subsystem, can be transmitted back to the earth by the telemetry system. Telemetry data is usually digitalized and sent as phase shift keying (PSK) of a low-power telemetry carrier with the help of time division techniques. The entire TDM frame might involve thousands of bits of data and take many seconds to transmit. The controlling earth station will be doing the function of monitoring, storing and decoding the data so that the state of any system or sensor within the satellite can be determined immediately by the system on the earth.

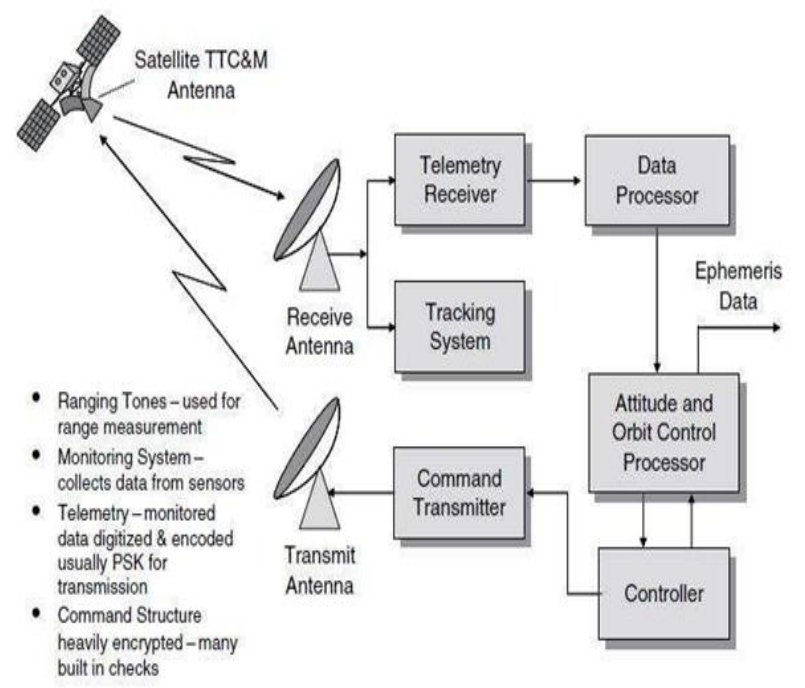

Fig 1: typical tracking, telemetry, command and monitoring system.

\section{TRACKING}

Many techniques can be used to know the current orbit of a satellite. The orbit'scurrent position can be determined by integrating the data obtained by velocity and acceleration sensors. The earth station controlling the satellite can monitor the Doppler shift of the telemetry carrier or beacon transmitter carrier to know the rate at which range is varying. The range determined from angular measurements from the earth station is capable of measuring the orbital elements .A pulse or a number of pulses is transmitted to the satellite and time delay before the pulse is received is monitored to calculate active range. Triangulation method is used to know the satellite's position when required number of earth stations is observing it. Measurements ranging can also be done using Ranging tones. A series of increasing frequency sine waves are given as an input to modulate the 
onboard generated carrier. The number of wavelengths of each frequency is determined by comparison of the phase of modulating component of sine wave. To determine the exact orbital position of the satellite, the elevation and azimuth angle measurements are most important.

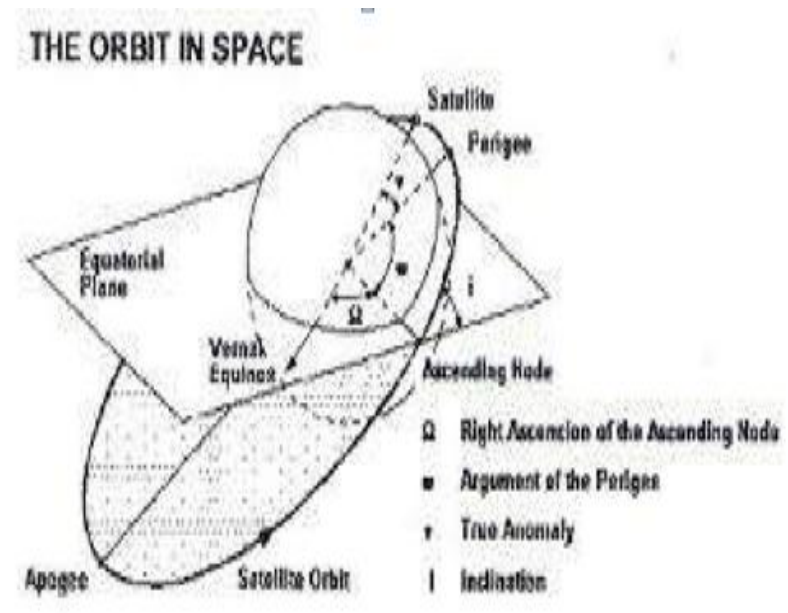

Fig 2: satellite in an inclined orbit

\section{COMMAND}

For the successful launch and operation of any communication satellite, a reliable and effective command structure is most needed. The correction to the orbit and changes in the altitude is performed by the command system. while launching, it is used to handle the firing of the apogee kick motor and to rotate a spinner or extend the solar sails and antennas of a three-axis stabilized satellite. Commands encryption and its responses is used to ensure the security in the command system. At the beginning the control code is converted into a command word and transmitted to the satellite in a TDM frame. The command and telemetry links can be able process in the same frequency band (6 and $4 \mathrm{GHz}$ ) but they are actually separated from the communication system. By any chance the main TTC \& M system fails, the backup system will keep the satellite on station. It is also ejects the satellite from geostationary orbit.

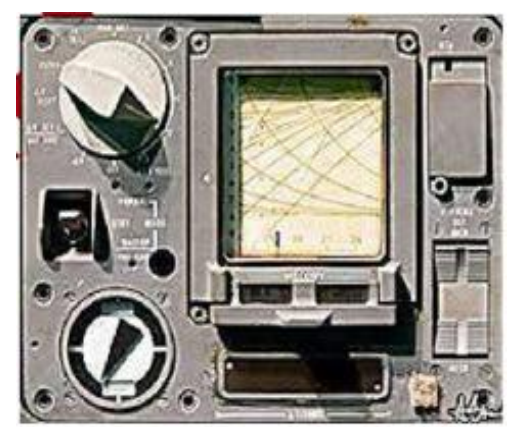

Fig 3: Control panel.

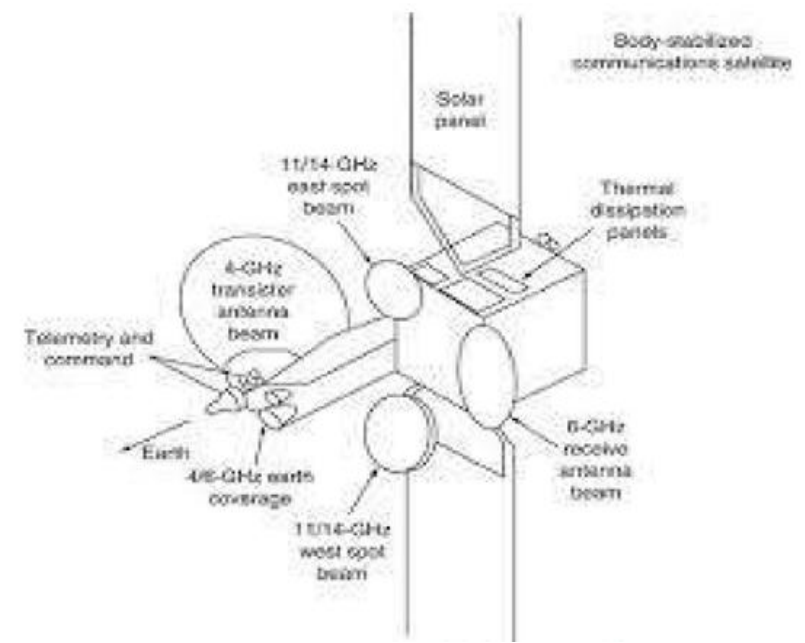

Fig 4: TTC \& M satellite.

\section{TT \& C INTERFACES}

\begin{tabular}{|l|l|}
\hline Subsystem & Requirement \\
\hline $\begin{array}{l}\text { Determination of altitude } \\
\text { and control }\end{array}$ & Antenna directing \\
\hline $\begin{array}{l}\text { Command handling and data } \\
\text { handling }\end{array}$ & $\begin{array}{l}\text { Command and telemetry } \\
\text { data rates Clock, bit sync } \\
\text { and requirements major } \\
\text { fault detection and its } \\
\text { recovery, Command and } \\
\text { telemetry electrical interface. }\end{array}$ \\
\hline Electrical Power Subsystems & $\begin{array}{l}\text { Distribution requirements } \\
\text { Thermal/Structural }\end{array}$ \\
$\begin{array}{l}\text { Heat sinks for TWTAs, Heat } \\
\text { dissipation for all active } \\
\text { boxes, Location of TT\&C } \\
\text { subsystem electronics, Clear } \\
\text { field of view for all antennas }\end{array}$ \\
\hline Payload & $\begin{array}{l}\text { Storing missing data, RF } \\
\text { and EMC } \\
\text { storagerequirements, Special } \\
\text { requirements for modulation } \\
\text { andcoding. }\end{array}$ \\
\hline
\end{tabular}

\section{TT \& C TRADE OFFS}

1. Antenna size vs transmitted power.

2. Solid state amplifiers vs travelling wave tube amplifiers.

3. Spacecraft complexity vs ground complexity.

\section{CONCLUSION}

On knowing the TTC\&M satellite system very well we concluded that the satellite should contain a number of subsystems to handle its communications mission. The altitude and orbital control system maintains the satellite in the preferred orbit. The Telemetry, Tracking, command and monitoring system allows the earth station to control the subsystems within the satellite and to monitor their status. Solar cells are required to generate the electrical power, batteries are often used for essential power during launch and eclipses. Satellites will eventually use the frequency reused that can allow the same RF spectrum to be used many 
times to increase the satellite's capacity. Antennas are the limiters in all radio communication system. An important issue in satelliteisreliability. Additional receiver and high power amplifier that can take over when a unit falls is provided by redundancy.

\section{REFERENCES}

[1] WALTER L. MORGAN, GARY D. GORDAN, Communication satellites handbook, Wiley Interscience, New York, 1989.

[2] TIMOTHY PRATT, CHARLES BOSTIAN, JEREMY ALLNUTT, Satellite Communications, second edition, Virginia Polytechnic Institute and State University, George Mason University, 2011(Reprint).

[3] W.H. BRAUN and J.E. KEIGLER, "RCA Satellite Networks: High Technology and Low User Cost,"Proceedings of the IEEE, 72, 1483-1505, November 1984.

[4] www.astrolink.com

[5] www.hns.com.spaceway

[6] Pisacane, Vincent L. and Robert C. Moore (1994); Fundamentals Of Space Systems, Oxford University Press, New York, 1994.

[7] Satellite communications system and apparatus- Paul Baron

[8] http://www.datatel-telemetry.de/

[9] https://en.wikipedia.org/wiki/AVS

[10] https://en.wikipedia.org/wiki/Space_tether 